\title{
Biosynthesis of Nickel Oxide Nanoparticles Using Fusarium verticillioides (Sacc.) and Their Biological Activity against Some Causative Agents of Mycotic Keratitis
}

\author{
S.A. El-Debaiky ${ }^{\#}$ A.S.M. El-Badry and M.M. El-Shahawy* \\ Botany Department and "Physics Department, Faculty of Science, Tanta University, \\ Tanta, Egypt.
}

\begin{abstract}
N THE present study, Nickel oxide (NiO) nanoparticles (NiONPs) were bio-synthesized extracellularly by the fungus Fusarium verticillioides (F. verticillioides) using Nickel Nitrate Hexahydrate $\mathrm{Ni}\left(\mathrm{NO}_{3}\right)_{2} \cdot 6 \mathrm{H}_{2} \mathrm{O}$ as a starting material. The formed NiONPs were precipitated in the form of a dark precipitate after adding the fungal filtrate to $\mathrm{Ni}\left(\mathrm{NO}_{3}\right)_{2} \cdot 6 \mathrm{H}_{2} \mathrm{O}$ solution. The properties of this formed precipitate were characterized by X-ray diffraction (XRD), transmission electron microscopy (TEM) and Fourier transformed infrared (FTIR). The XRD pattern indicated that the NiONPs had a face-centered cubic (FCC) structure and their average crystallite size was found to be $8-17.7 \mathrm{~nm}$. The average particle size found to be $8.5-20.7 \mathrm{~nm}$ from TEM image, which was compatible with XRD result. The FTIR analysis bands revealed the chemical composition bonding of the formed nano-NiO compound.
\end{abstract}

The antifungal activity of the formed NiONPs was tested against some human pathogenic fungi which were isolated from mycotic keratitis patients, inpatient department of ophthalmology hospital, Tanta University, Egypt. The best results obtained against Candida albicans, $C$. tropicalis and Aspergillus niger where the minimum inhibitory concentration (MIC) was $12.5,25$ and $25 \mathrm{mg} / \mathrm{ml}$, respectively. Further confirmation of NiONPs biological activity was employed by measurement of their concentration at different intervals within skin, blood and liver of experimental animals, showing a promising absorption and washing out rates from mice tissues; these results indicated that the NiONPs did not accumulated within mice bodies.

Keywords: Nickel oxide, Nanoparticles, Fusarium verticillioides, TEM, XRD, Mycotic keratitis, MIC.

\section{Introduction}

In the last few decades, many researchers were interested in preparing and studying the nanostructured materials that have unique physical and chemical properties. These properties are dependent on size, shape and crystallography of nanomaterials. Therefore, the synthesis of nanostructured materials with controlled size and shape becomes very important (Al-Sehemi et al., 2014). Currently, unlike the chemical and physical synthetic methods, biological systems provide a novel idea for the production of nano-materials. Several plant extracts and even microorganisms from bacteria to fungi were utilized to synthesize inorganic materials in nano-scale either intra- or extracellularly (Sagar \& Ashok, 2012).

Fungi have several advantages over other microorganisms for the biosynthesis of nanoparticles since most species are easy to handle, require simple nutrients, possess a high cell wall-binding capacity and high intracellular metal uptake capabilities (Salvadori et al., 2015). Also fungi could be utilized for a clean, nontoxic, and environmentally acceptable "green" biosynthesis of metals nanoparticles. Many fungal species have been used in this process, including Fusarium oxysporum (Soni \& Prakash, 2012).

$\mathrm{NiO}$ is considered one of the most commonly used metal oxides for a wide range of applications where NiONPs were shown to have novel optical, electronic, magnetic, thermal, and mechanical properties. In addition, it was used in many potential applications such as in battery electrodes, gas sensors, photo-electronic devices....etc. (Gondal et al., 2012; Qiao et al., 2009 and Salavati-Niasari et al., 2009). 
Recently, several methods have been developed for the synthesis of NiONPs, such as ultrasonic radiation, hydrothermal synthesis, carbonyl method, and laser chemical method, pyrolysis by microwave, sol-gel method, coprecipitation and microemulsion method. On the other hand, the NiONPs were biosynthesized using dead biomass of the filamentous fungus Hypocrea lixii.(Rahdar et al., 2015 and Salvadori et al., 2015)

Human mycosis can be defined as a disease caused by fungi, causing inflammations, and many tissue disorders in different sites within the human body, and thus becoming an important constituent of worldwide public health problems (Hunter \& Strickland, 2000). Ocular mycosis can be defined as the pathogenic growth of fungi within different eye tissues. It can be grouped according to the origin, and site of infection into 3 types. First type is mycotic keratitis which is a superficial secondary infection of the injured cornea. Second type is endogenous ocular mycosis results from dissemination of other systemic fungal infections into the orbit through the adjacent tissues. Third type is miscellaneous ocular mycosis that invades tear ducts, eyelids and conjunctiva (Shukla \& Singh, 1997). Human keratitis is an inflammation of the cornea, the transparent part of the eye covering the iris and pupil, and has distinct transparency to allow accurate vision and passage of light. Migration of the inflammatory cells as an immune response into the corneal layers can disrupt the corneal transparency, resulting in corneal opacification or complete blindness. Severity of keratitis depends on the number of migrating inflammatory cells; and have viral, bacterial, acanthamoebal or fungal origin (Djalilian et al., 2001). The number of mycotic keratitis cases aggressively increased all over the world due to microbial imbalance, and disturbed ecological relationships among human, and surrounding microorganisms (Wong et al., 1997).

The present study aimed to achieve the biosynthesis and characterization of the single phase NiONPs extracellularly using the mycelial filtrate of the fungus $F$. verticillioides. Also, the antifungal activity of the biosynthesized NiONPs was tested against fungal isolates causing human mycotic keratitis at Tanta University Hospital.

\section{Materials and Methods}

Tested fungus and chemical preparations

For the present study, culture of $F$. verticillioides was kindly provided by faculty of agriculture, Mansoura University, Egypt. The fungus was maintained on potato dextrose agar medium (PDA). Nickel nitrate $\mathrm{Ni}\left(\mathrm{NO}_{3}\right)_{2} \cdot 6 \mathrm{H}_{2} \mathrm{O}$ was used as the starting material for the biosynthesis of NiONPs. It was purchased from Oxford Laboratory Company, India.

\section{Used media \\ "Potato dextrose liquid medium" (PDL)}

The medium has the following constituents (g/liter), potato tubers 200, D-Glucose, 20. Tubers of potatoes were peeled, cut to small pieces and boiled for about one hour in one liter of distilled water. The potato pieces were mashed and squeezed through a fine sieve and thereafter glucose was added. The mixture volume was adjusted to one liter with distilled water. A 500 mg capsule of chloramphenicol antibiotic was added as antibacterial agents for preventing the bacterial contamination. The medium then was sterilized and stored for further work (Moubasher, 1993).

\section{"Sabouraud's dextrose agar" (SDA)}

The medium has the following constituents (g/liter), peptone, 10, D-Glucose, 20 and $2 \%$ Agar (Moubasher, 1993).

\section{Preparation of biomass and mycelial filtrate of $F$. verticillioides}

The method adopted to Hussein (2016) and Shi et al. (2015) was performed. The fungal biomass of $F$. verticillioides for NiONPs biosynthesis, was grown aerobically in PDL medium. After inoculation with $F$. verticillioides, the flasks were incubated in an orbital shaker at $30 \pm 2^{\circ} \mathrm{C}$ and agitated at $150 \mathrm{rpm}$ for 3-4 days. After the incubation period, the fungal biomass was harvested using a sieve composed of cloth with very narrow pores. The fungal biomass was washed with distilled water to remove any medium components. Typically, $15 \mathrm{~g}$ (wet weight) of fungal biomass were soaked in $150 \mathrm{~mL}$ sterile distilled water then agitated in an orbital shaker at $30 \pm 2^{\circ} \mathrm{C}$ for 3-4 days. The mycelial filtrate was prepared by removing the fungal mycelia from the flasks after 3-4 days using double ring filter paper No.201. The resulted filtrate was centrifuged at $3500 \mathrm{rpm}$ for $10 \mathrm{~min}$ to remove the Fusarium spores. The filtrate of $F$. verticillioides was clear and ready for the biosynthesis of NiONPs. 
Biosynthesis of NiONPs using the mycelial filtrate of $F$. verticillioides

Fifty milliliters of $0.001 \mathrm{M} \mathrm{Ni}\left(\mathrm{NO}_{3}\right)_{2} \cdot 6 \mathrm{H}_{2} \mathrm{O}$ solution were prepared; then added to $800 \mathrm{ml}$ of the fungal filtrate drop wise with continuous stirring for one hour until appearing of brown turbidity in the green solution of $\mathrm{Ni}\left(\mathrm{NO}_{3}\right)_{2} \cdot 6 \mathrm{H}_{2} \mathrm{O}$. This turbidity indicates the formation of NiONPs, which was precipitated at the bottom by stopping stirring and keeping the solution stable overnight. The ppt. was then centrifuged at $4000 \mathrm{rpm}$ for 10 min and washed with distilled water and allowed to dry at room temperature. The air-dried ppt. was heated at $200^{\circ} \mathrm{C}$ for $12 \mathrm{~h}$ to remove any remaining nitrate, and then annealed at $400^{\circ} \mathrm{C}$ and $600^{\circ} \mathrm{C}$ for 2 h (Mamuru et al., 2015).

\section{Characterization of $\mathrm{NiONPS}$}

The X-ray diffraction (XRD) of nanoparticles was carried out at room temperature using APD 2000 pro, H423-virtical diffractometer equipped with $\mathrm{Cu} \mathrm{K} \alpha$ radiation $(\lambda=1.540598 \AA)$. The particle size and morphology of NiONPs were investigated by JEOL JEM-2100 transmission electron microscopy (TEM).The TEM specimen was prepared by the ultrasonic dispersion of small amount of the powders in a few milliliters of ethanol, and then dropping on a copper grid covered in an amorphous carbon film. Fourier transformed infrared spectroscopy (FTIR) spectra were carried out at room temperature using a BRUKER FT-IR spectrometer tensor 27 on the range 200 to $4000 \mathrm{~cm}^{-1}$ at Central Lab., Tanta University.

\section{Collection and identification of pathogenic fungal isolates}

Fungal isolates were collected from infected human eyes during regular once a week visits to the outpatient clinic, and daily follow up visits for patients admitted to inpatient department of ophthalmology hospital, Tanta University, Egypt. Samples were collected during the summer season (July - September) of 2016 from 50 patients that were clinically diagnosed by the physician's team of the clinic to possess mycotic corneal ulcers. All samples were collected by flamed "Kummra" platinum spatula (Alcon-Couvreur, Belgium) for hard tissues, or by ethylene gas-sterilized single use cotton swab (BioMed, China) for soft ulcers to avoid perforation. Aseptic conditions of sampling were considered by avoiding conjunctiva and eye lids during corneal scraping; betadine (Nile Pharma, Cairo, Egypt.) was applied as a gentle washing around infected eyes; and samples were isolated in a closed controlled room in the hospital laboratory. Then $\mathrm{C}$-streaks were made on culture plates to distinguish microbial growth of corneal scraping from other plate contaminants (Margo \& Brinser, 1987). Each sample was cultured on 2 sterile Petri dishes with sterile SDA medium, one plate was incubated at $27^{\circ} \mathrm{C}$ for 3 days, and the other plate was incubated at $37^{\circ} \mathrm{C}$ for $48 \mathrm{~h}$. Fungal colonies were subcultured for purification, and identification on the same medium under the same incubation conditions.

Isolated filamentous fungi were identified morphologically by examination under light microscope at $400 \mathrm{X}$. The identification process was according to Barnett \& Hunter (1998) for the genera of fungi imperfecti (Ramirez, 1982) for Penicillia and Raper \& Fennell (1965) for Aspergilli. Suspected colonies of yeast were streaked onto SDA plates, and incubated for $48 \mathrm{~h}$ at $37^{\circ} \mathrm{C}$. These cultures were used as the source of inoculum for the yeast confirmatory tests as the isolated yeasts were identified according to Larone (2011) using API 20 CAUX from Biome' rieux SA (France).

\section{Incidence and predisposing factors of mycotic keratitis}

For each patient, much information was collected to analyze his circumstancing conditions as follows: age, gender, family crowding, residence conditions, occupational career and medical history.

Screening for antifungal activity of prepared NiONPs against the collected pathogenic isolates

Cut plug method recorded by Pridham et al. (1956) was employed to determine the antifungal activity of the prepared suspension of NiONPs as follows: freshly prepared cell/spore suspension of the collected fungal strains $(0.5 \mathrm{ml}$ of about $10^{6}$ cells $\mathrm{ml}^{-1}$ ) was mixed with $9.5 \mathrm{ml}$ of melting sterile SDA medium, poured on sterile Petri dishes, and left to solidify at room temperature. Regular wells were made in the inoculated agar plates by a sterile cork borer of $0.7 \mathrm{~mm}$ diameter. Each well was filled with $100 \mu \mathrm{L}$ of $(1 \mathrm{~g} / 10 \mathrm{ml})$ NiONPs suspension. Three replica were made for each test, and all plates were incubated at $27^{\circ} \mathrm{C}$ for 3 days for filamentous fungi, and at $37^{\circ} \mathrm{C}$ for $48 \mathrm{~h}$ for yeast, then the average diameters of inhibition zones were recorded in millimeters. 


\section{Determination of minimal inhibitory concentration (MIC) of NiONPS}

Serial dilutions were made to prepare concentrations of $100,50,25,12.5$ and 6.25 $\mathrm{mg} \mathrm{ml} \mathrm{m}^{-1}$ of the aqueous suspension of the tested NiONPs preparation; zero concentration was considered as a negative control of the solvent, that was considered as the control growth. A previously prepared pure cell/spore suspension of the most affected fungal pathogenic isolate $(0.5 \mathrm{ml}$ of about $10^{6}$ cells $\mathrm{ml}^{-1}$ ) was mixed with $9.5 \mathrm{ml}$ of each concentration in sterile test tubes, incubated at $27^{\circ} \mathrm{C}$ for 3 days for filamentous fungi, and at $37^{\circ} \mathrm{C}$ for $48 \mathrm{~h}$ for yeast; then optical density of growth was measured by spectrophotometer (Optima SP300 , Japan) at $620 \mathrm{~nm}$ for each incubated mixture. Results were represented graphically, and MIC was recorded (Shadomy et al., 1985).

\section{Persistence and absorption of NiONPs in experimental animal tissues}

Eight weeks old Swiss albino female mice weighing 21-23 grams were purchased from the animal house at National Research Center (NRC), Giza, Egypt. Mice were bred in the animal house of faculty of science, Tanta University. They were maintained under a $12 \mathrm{~h}$ light dark cycle at a temperature of $22 \pm 2^{\circ} \mathrm{C}$ and fed with standard diet and water. The study was conducted according to the ethical norms approved by the animal ethics committee guide lines in faculty of science, Tanta University. All the mice were divided into 2 groups, each has 9 animals (3 intervals $\times 3$ replica): one control group of three non-treated animals for standardization of all records and blanks; the other group was subjected to regular scratches on hair-removed dorsal skin of $2^{\times} 2 \mathrm{~cm}$ lesion, washing lesions with NiONPs suspension of $25 \mathrm{mg} / \mathrm{ml}$ each $2 \mathrm{~h}$ regular intervals for $24 \mathrm{~h}$. Control and treated mice were incubated, and then 3 mice of each group were sacrificed by cervical dislocation after 12, 24 and 48h. The skin, blood, spleen and liver samples were collected from the mice; all samples were macerated separately in sterile aqueous solution and standardized at conc. of $1 \mathrm{~g}$ tissue / $10 \mathrm{ml}$ dist. $\mathrm{H}_{2} \mathrm{O}$, centrifuged at 3000 rpm, then light absorbance of all supernatants was measured at $395 \mathrm{~nm}$; concentration of NiONPs was determined according to a previously prepared standard curve (Lasagna-Reeves et al., 2010).

\section{Statistical analysis}

Statistical analysis of the present study was conducted, using the mean, standard deviation and F-value using the computer programme IBM SPSS Statistics 19.

Results and Discussion

Optical Property

The appearance of brown ppt. in the green solution of $\mathrm{Ni}\left(\mathrm{NO}_{3}\right)_{2} \cdot 6 \mathrm{H}_{2} \mathrm{O}$ after adding the fungal filtrate and let it stable overnight indicates the formation of NiONPs (Fig.1).

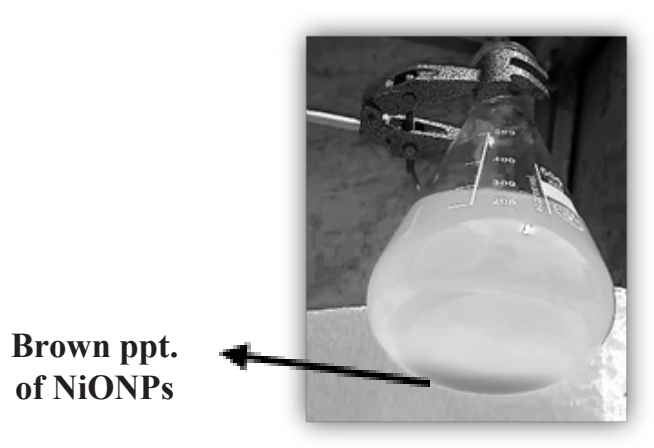

Fig.1. Brown ppt. of NiONPs

\section{$X$-Ray diffraction studies}

Figure.2 ( $a, b$ and $c$ ) shows the XRD patterns of the produced NiONPs; as prepared, after annealing at $400^{\circ} \mathrm{C}$ and $600^{\circ} \mathrm{C}$, respectively. The XRD of NiONPs shows the existences of reflection peaks 111, 220, 311,222 and $100 \%$ peak intensity observed from the plane 200. These peaks are clearly indicating that the NiONPs phase exist in the form of face centered cubic (FCC) of polycrystalline structure (ICSD Card No. 01-075-0269).This result is mostly agreed with another recent study (El-Nahass et al., 2015). After annealing at $400^{\circ} \mathrm{C}$ for $2 \mathrm{~h}$, broad diffraction peaks appears while these peaks become sharply after annealing at $600^{\circ} \mathrm{C}$ for $2 \mathrm{~h}$ which indicating the increase of the crystallinity of NiONPs.

The structural parameters of the produced NiONPs after annealing at $400^{\circ} \mathrm{C}$ and $600^{\circ} \mathrm{C}$ are shown in Table.1. These structure parameters including lattice constant $\alpha$ and the crystallite size D. The inter planar distance was calculated using Bragg's Law, and lattice constant of the cubic unit cell of the NiONPs is determined from the following relation:

$$
a=d_{h k l} \sqrt{h^{2}+k^{2}+l^{2}}
$$

where: $d_{h k l}$ : The inter planar spacing of the atomic planes (d-spacing). and $\mathrm{h} \mathrm{k} \mathrm{l}$ : Miller indices of each plane. 


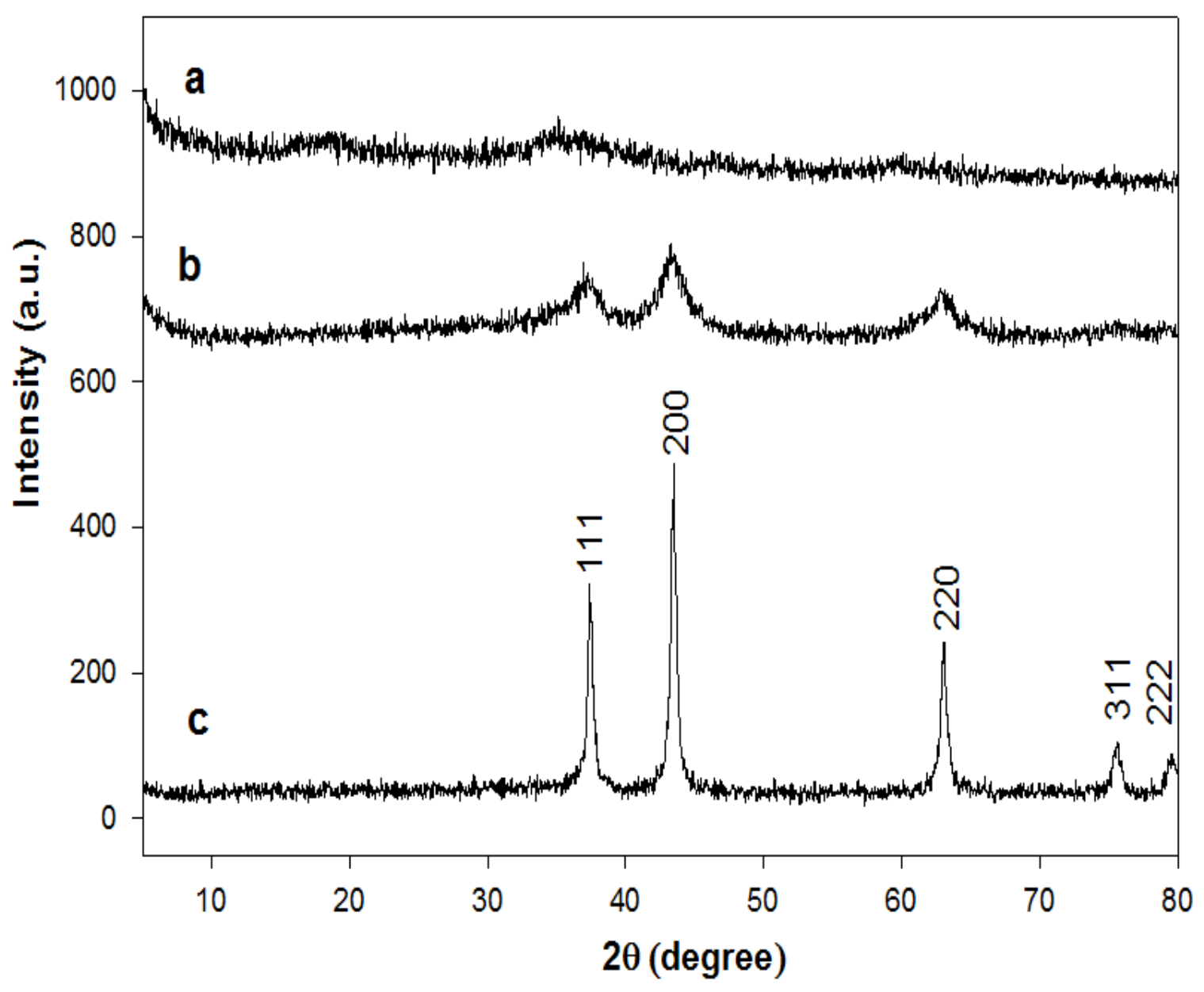

Fig. 2. XRD pattern of NiONPs

(a) as prepared, (b) after annealing at $400^{\circ} \mathrm{C}$ and (c) after annealing at $600^{\circ} \mathrm{C}$

TABLE.1. The structural parameters of NiONPs.

\begin{tabular}{|c|c|c|c|c|c|c|c|c|c|c|}
\hline \multirow[t]{2}{*}{ ( h k l) } & \multicolumn{2}{|c|}{ d-spacing d $(\AA)$} & \multicolumn{2}{|c|}{$\begin{array}{c}\text { Diffraction angle } \\
2 \theta \text { (degree) }\end{array}$} & \multicolumn{2}{|c|}{$\begin{array}{l}\text { Relative intensity } \\
\qquad \mathbf{I} / \mathbf{I}_{0}\end{array}$} & \multicolumn{2}{|c|}{$\begin{array}{c}\text { Lattice constant } \\
\text { a ( }(\AA)\end{array}$} & \multicolumn{2}{|c|}{$\begin{array}{c}\text { Crystallite size D } \\
\text { (nm) }\end{array}$} \\
\hline & $400^{\circ} \mathrm{C}$ & $600^{\circ} \mathrm{C}$ & $400^{\circ} \mathrm{C}$ & $600^{\circ} \mathrm{C}$ & $400^{\circ} \mathrm{C}$ & $600^{\circ} \mathrm{C}$ & $400^{\circ} \mathrm{C}$ & $600^{\circ} \mathrm{C}$ & $400^{\circ} \mathrm{C}$ & $600^{\circ} \mathrm{C}$ \\
\hline (111) & 2.4118 & 2.4025 & 37.25 & 37.4 & $75.3 \%$ & $63 \%$ & 4.177 & 4.1613 & 6.967 & 19.52 \\
\hline$(200)$ & 2.0862 & 2.078 & 43.34 & 43.5 & $100 \%$ & $100 \%$ & 4.172 & 4.156 & 8.436 & 21.14 \\
\hline$(220)$ & 1.4765 & 1.472 & 62.89 & 63.1 & $68.4 \%$ & $50 \%$ & 4.176 & 4.163 & 8.168 & 16.76 \\
\hline (311) & 1.2579 & 1.257 & 75.5 & 75.6 & $13.7 \%$ & $14 \%$ & 4.172 & 4.169 & 8.576 & 14.2 \\
\hline$(222)$ & - & 1.203 & - & 79.6 & - & $12 \%$ & - & 4.1687 & - & 17.04 \\
\hline & & & average & & & & 4.174 & 4.164 & 8.037 & 17.73 \\
\hline
\end{tabular}


The average crystallite size (D) of NiONPs is calculated using the Scherer's equation as following Theivasanthi \& Alagar (2012):

$$
D=\frac{0.9 \lambda}{h_{1 / 2} \cos \theta_{B}}
$$

where: $\lambda$ : The wavelength for $\mathrm{Cu}-\mathrm{K} \alpha(\lambda=1.540598 \AA)$.

$\boldsymbol{h}_{1 / 2}$ : The full width at half maximum (FWHM) of the diffraction peak. and

$$
\boldsymbol{\theta}_{B} \text { : The diffraction angle. }
$$

Using the obtained XRD analysis pattern (Fig.2 b and c) and Scherer's equation, the average crystalline size of the produced NiONPs was calculated and found to be $8 \mathrm{~nm}$ and $17.7 \mathrm{~nm}$ for the prepared samples annealed at $400^{\circ} \mathrm{C}$ and $600^{\circ} \mathrm{C}$, respectively (Table. 1 ).

\section{The transmission electron microscopy (TEM)}

The transmission electron micrographs of the produced NiONPs after annealing shown in Fig.3. Accordingly, the majorities of the NiONPs are relatively uniform in diameter where the observed average particle size found to be about $8.5 \mathrm{~nm}$ for sample annealed at $400^{\circ} \mathrm{C}$ (Fig.3a) while it was $20.7 \mathrm{~nm}$ when the sample annealed at $600^{\circ} \mathrm{C}$ (Fig.3b). This is agreement with the average particle size that calculated using Scherrer's equation and XRD line broadening studies. Other studies also synthesized the NiONPs with different particle size in nano range using different methods. Using the solid-state thermal decomposition method and the anodic arc plasma method, the particle size of NiONPs was $15 \mathrm{~nm}$ and 15-45 nm, respectively (Barakat et al., 2013 and Qiao et al., 2009). While the average particle size of biosynthesized NiONPs using the dead biomass of the fungus Hypocrea lixii was 3.8 and $1.25 \mathrm{~nm}$ (Salvadori et al., 2015) which consider a better method to produce nanoparticles.

\section{The Fourier transformed infrared (FTIR)}

The FTIR spectra at room temperature in the range of (200-4000) $\mathrm{cm}^{-1}$ for the annealed NiONPs samples at $400^{\circ} \mathrm{C}$ and $600^{\circ} \mathrm{C}$ are shown in Fig.4.The absorption band at $439 \mathrm{~cm}^{-1}$ is assigned to $\mathrm{Ni}-\mathrm{O}$ stretching mode, which is a clear evidence for the presence of the crystalline $\mathrm{NiO}$ (Gondal et al., 2012; Rahdar et al., 2015 and Salavati-Niasari et al., 2009). The broadness of the absorption band indicates that the $\mathrm{NiO}$ powders are nanocrystals (Qiao et al., 2009). The absorption bands at 555 $\mathrm{cm}^{-1}$ and $3389 \mathrm{~cm}^{-1}$ attributed to Ni-O-H and $\mathrm{O}-\mathrm{H}$ stretching bonds, respectively (Rahdar et al., 2015).

Collection and identification of pathogenic fungal isolates

Seven fungal isolates were obtained from 50 patients during the present survey from Ophthalmology Hospital, Tanta University, Egypt during summer, 2016. Patients were clinically diagnosed to have different type of corneal ulcers and fungal infections were confirmed by culture findings; whereas the represented yeast forms were Candida albicans, Candida pelliculosa, C.tropicalis and Stephanoascus ciferrii; the resultant filamentous fungi were Aspergillus niger, Penicillium marneffei and Cladosporium carrionii as shown in Table.2.

These findings were in agreement with the records of Abu El-Souod et al.(2013), El-Badry \& Ali (2015) and Mahmoud et al. (2004), who stated that the most dominant fungal pathogens isolated from mycotic keratitis were Aspergillus flavus, A.niger, Penicillium lanosum, Fusarium moniliforme and Cladosporium sphaerospermum.

Incidence and predisposing factors of mycotic keratitis

Predisposing factors were recorded and summarized in Table. 3 regarding the spread of mycotic keratitis in the present survey as follows: Mycotic ulcers were observed to be common among patients aged more than 40 years old ( 37 cases $=74 \%$ out of total mycotic ulcers), and lowered to be only 13 cases $(26 \%)$ for patients of less than 40 years old. It is obviously noticeable that mycotic keratitis was more common in males during the present survey $(29$ cases $=58 \%)$ more than females $(21$ cases $=42 \%)$. Another important risk factor affecting the spread of mycotic keratitis was the family crowding index, it was observed that mycotic ulcers recorded higher rates in families of crowding index $>4$ persons ( 34 cases $=68 \%$ ), than families of crowding index $\leq 4$ persons ( 16 cases $=32 \%$ ). Social hygiene parameters had a great effect on the spreading of infectious human mycotic corneal ulcers. Patients holding rural houses possessed higher incidence of mycotic ulcers (33 cases $=66 \%$ ), more than those in urban houses ( 17 cases $=34 \%$ ). On the other hand, the higher incidence of mycotic ulcers (34 cases $=68 \%$ ) among patients having outdoor pipes water source than indoor pumps $(16$ cases $=32 \%)$ and sewage disposal by conservancy $(31$ cases $=62 \%)$ than closed pipe sanitation $(19$ cases $=38 \%)$. 

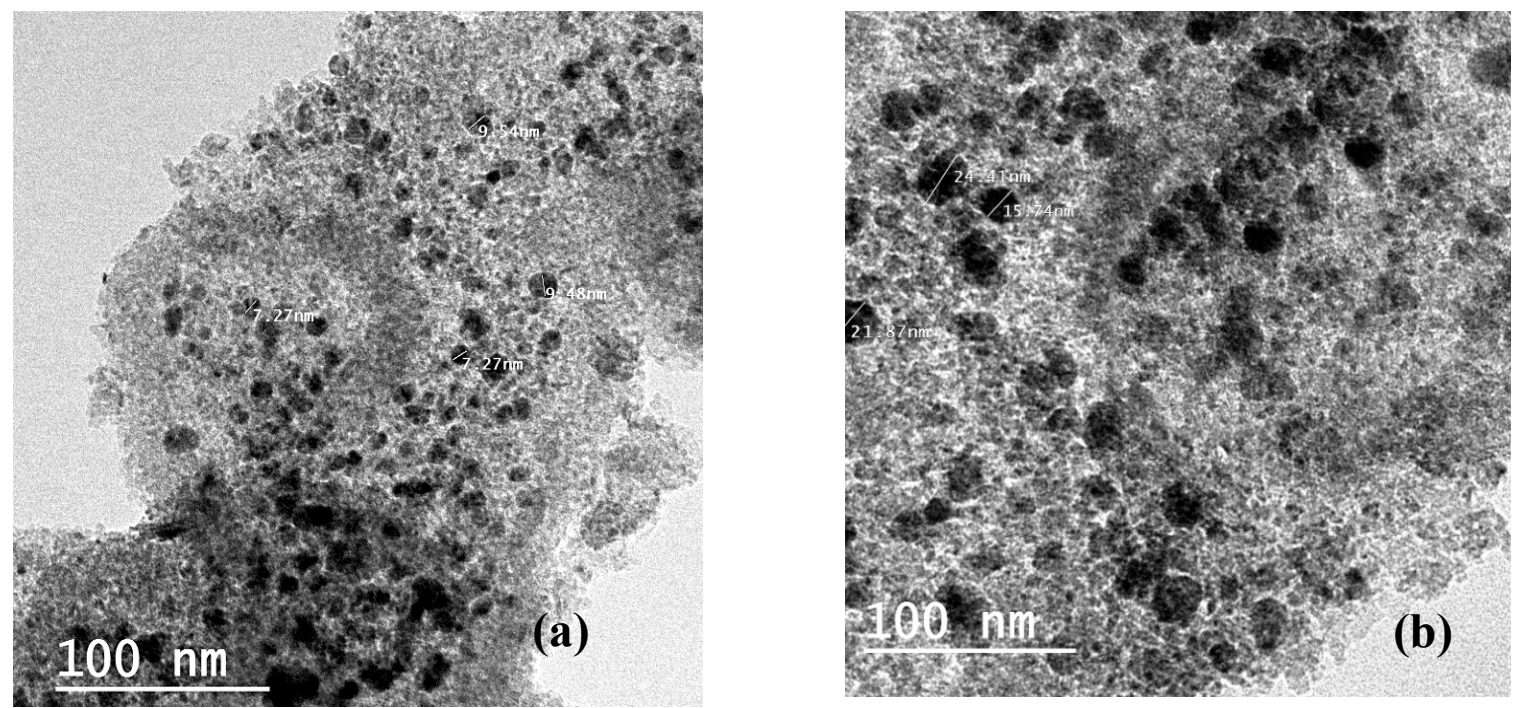

Fig . 3.TEM images of annealed NiONPs (a) at $400^{\circ} \mathrm{C}$ and (b) at $600^{\circ} \mathrm{C}$.

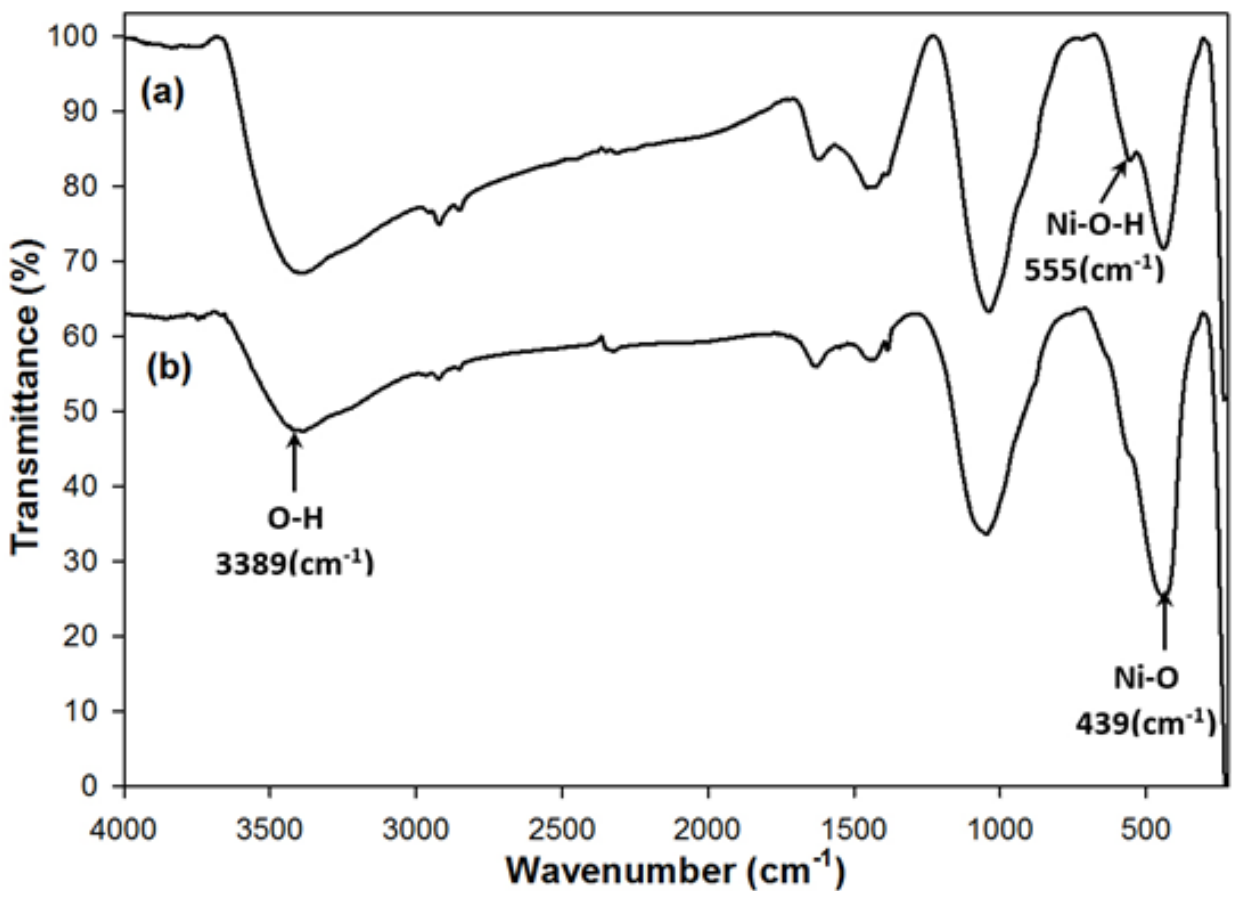

Fig.4. FTIR of annealed NiONPs (a) at $400^{\circ} \mathrm{C}$ and (b) at $600^{\circ} \mathrm{C}$.

TABLE 2. Incidence of yeasts and filamentous fungi in infected eyes during summer, 2016.

Yeasts species

1- Candida albicans Berkhout, 1923

2- Candida pelliculosa Redaelli, 1925

3- Candida tropicalis Berkhout, 1923

4- Stephanoascus ciferrii Smith, 1976
Filamentous fungi species

1- Aspergillus niger Tieghem, 1867

2- Penicillium marneffei Segretain, 1959

3- Cladosporium carrionii Link, 1816 
TABLE 3. Predisposing factors affecting distribution of human mycotic keratitis during summer, 2016.

\begin{tabular}{|c|c|c|c|}
\hline Parameter & Category & No. of cases & $\begin{array}{c}\text { Percentage out } \\
\text { of total cases }\end{array}$ \\
\hline \multirow[t]{3}{*}{ Age } & $\leq 40$ years & 13 & 26 \\
\hline & $>40$ years & 37 & 74 \\
\hline & Total & 50 & 100 \\
\hline \multirow[t]{3}{*}{ Gender } & Male & 29 & 58 \\
\hline & Female & 21 & 42 \\
\hline & Total & 50 & 100 \\
\hline \multirow[t]{2}{*}{ Family crowding index } & $<4$ & 16 & 32 \\
\hline & $\geq 4$ & $\begin{array}{l}34 \\
50\end{array}$ & $\begin{array}{c}68 \\
100\end{array}$ \\
\hline \multirow[t]{3}{*}{ Type of house } & Rural & 33 & 66 \\
\hline & Urban & 17 & 34 \\
\hline & Total & 50 & 100 \\
\hline \multirow[t]{3}{*}{ Water source } & Indoor pipes & 16 & 32 \\
\hline & Outdoor pumps & 34 & 68 \\
\hline & Total & 50 & 100 \\
\hline \multirow[t]{3}{*}{ Sewage disposal } & Closed pipes & 19 & 38 \\
\hline & Conservancy & 31 & 62 \\
\hline & Total & 50 & 100 \\
\hline
\end{tabular}

These results were confirmed by the study of Moharram et al. (1999), who revealed that fungal keratitis affected males more than females, and was more frequent in those aged from 20 to 50 years. Other worldwide surveys for mycotic keratitis confirmed the role of Aspergillus spp., Fusarium spp. and Candida spp. in corneal infections and ulcers and their spread among patients aged from 20 to 60 years with low social hygienic parameters, as stated by Leck et al. (2002) in Ghana, Thomas \& Kaliamurthy (2013) in UK and Sun et al. (2007)in USA.

Screening for antifungal activity of prepared NiONPS against the collected pathogenic isolates and determination of MIC of NiONPS

The NiONPs suspension that was prepared in the present study with the aid of fungal biosynthesis, was observed to possess a promising antifungal activity against the infectious fungal isolates obtained from mycotic keratitis cases; as the most affected isolate was Candida albicans with inhibition zone diameter $=26 \mathrm{~mm}$, followed by Candida pelliculosa and Aspergillus niger (inhibition zone diameters $=24$ and $21 \mathrm{~mm}$, respectively), whereas the lowest antifungal effect was recorded for Cladosporium carrionii $(12 \mathrm{~mm})$ as represented in Table.4 in vitro.
TABLE 4. Preliminary survey for antifungal activity of the prepared NiONPs against the isolated pathogens

\begin{tabular}{lc}
\hline Tested isolate & $\begin{array}{c}\text { Inhibition } \\
\text { Zone (mm) } \\
\text { (Mean } \pm \text { SD) }\end{array}$ \\
\hline Candida albicans & $26 \pm 3$ \\
Candida pelliculosa & $13 \pm 2$ \\
Candida tropicalis & $24 \pm 1$ \\
Stephanoascus ciferrii & $14 \pm 2$ \\
Aspergillus niger & $21 \pm 1$ \\
Penicillium marneffei & $16 \pm 1$ \\
Cladosporium carrionii & $12 \pm 3$ \\
F-value & $3.136^{*}$ \\
\hline
\end{tabular}

SD: Standard deviation *: Significant at $\mathrm{P} \leq 0.044$

The MIC of NiONPs suspension was identified as the first concentration at which a stable number of surviving cells was recorded against the most affected fungal isolates in the present survey with very low ratio with no increasing effect for the higher concentrations, that was recorded at the range of $12.5-25 \mathrm{mg} / \mathrm{ml}$ in vitro, as revealed from Fig.5. 


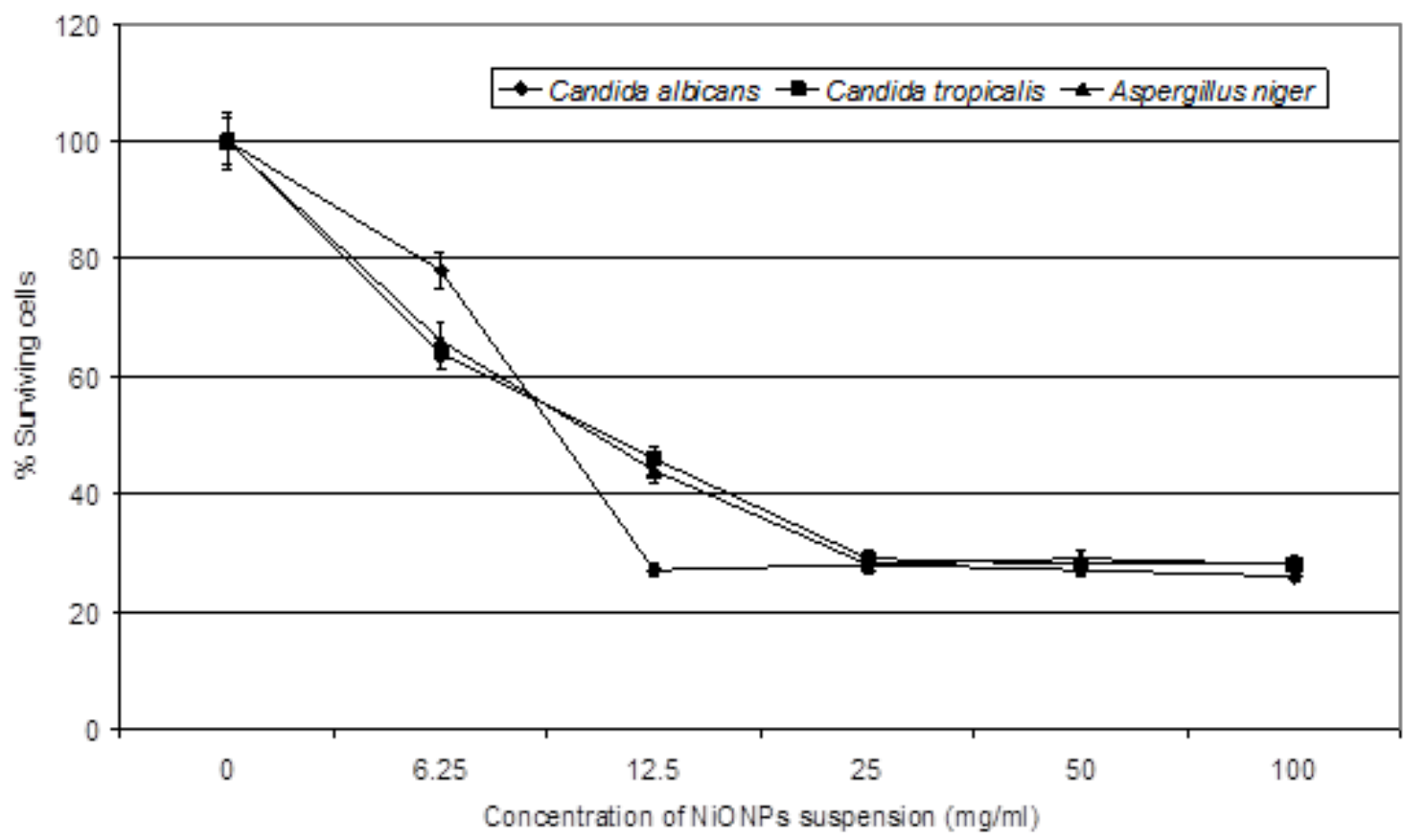

Fig.5.The MIC of NiONPs against the most affected isolates.

NiONPs offer a great possibility for biomedical application, not only to deliver pharmaceutics, but also to be used as novel diagnostic and therapeutic approaches. The small sizes of NPs imply that they could get close to a biological target of interest more effective penetration and absorption inside living tissues.Considering the growing interest in nanomedicine, the observation of nanoparticle accumulation in the skin is significant from a number of perspectives. The ability to use skin to determine exposure provides a non-invasive and rapid determination of nanoparticle exposure that can be further quantified to determine the range of NPs absorption of washing out for the internal tissues of experimental animals to evaluate the safety of medical use of NPs and the level of their accumulation in internal tissues (Elsaesser \& Howard, 2012).

\section{Persistence and absorption of NiONPs in experimental animal tissues}

As a confirmation for the biological activity of the prepared NiONPs in vivo, their penetration, absorption and persistence within the experimental animal tissues were investigated in Table.5. Relatively adequate amounts of NiONPs were established within skin layer after $12 \mathrm{~h}$ of topical administration of their aqueous suspension on the scratched skin $(236 \mu \mathrm{g} / \mathrm{ml})$, that was washed out and decreased to $76 \mu \mathrm{g} / \mathrm{ml}$ after $48 \mathrm{~h}$. Moderate rate of NiONPs into the blood stream was recorded after $12 \mathrm{~h}$, that was washed out to a very low amount $(12 \mu \mathrm{g} / \mathrm{ml})$ after $48 \mathrm{~h}$. These findings were supported by the same pattern of NiONPs absorption and washing out within liver tissue, that was accumulated to $72 \mu \mathrm{g} / \mathrm{ml}$ after $24 \mathrm{~h}$, and then washed to only $15 \mu \mathrm{g} / \mathrm{ml}$ after 48 $\mathrm{h}$; with a promising strategy for antifungal activity of NiONPs, possessing a noticeable washing rate from experimental animal tissues.

TABLE 5. Accumulation of NiONPs in experimental animal tissues.

\begin{tabular}{cccc}
\hline $\begin{array}{c}\text { Penetrated } \\
\text { tissue }\end{array}$ & \multicolumn{3}{c}{ Concentration of NiONPs $(\boldsymbol{\mu g} /$} \\
& \multicolumn{3}{c}{$\mathbf{m l}) \pm$ SD } \\
& After & After & After \\
& $\mathbf{1 2} \mathbf{~ h}$ & $\mathbf{2 4} \mathbf{~ h}$ & $\mathbf{4 8 ~ h}$ \\
\hline Skin & $236.33 \pm$ & $161 \pm 9$ & $\mathbf{7 6 . 6 7} \pm$ \\
& 14.5 & & $\mathbf{4 . 0 4}$ \\
Blood & $114.33 \pm$ & $44.33 \pm$ & $\mathbf{1 2} \pm \mathbf{1}$ \\
& 4.5 & 3.5 & \\
Liver & $23 \pm 2$ & $73 \pm 5.56$ & $\mathbf{1 5} \pm \mathbf{2}$ \\
Spleen & $\mathbf{0}$ & $\mathbf{0}$ & $\mathbf{0}$ \\
F-value & $588.884^{*}$ & $445.515^{*}$ & $667.516^{*}$ \\
\hline
\end{tabular}

SD: Standard deviation *: Significant at $\mathrm{P} \leq 0.000$ 
These findings revealed that the tested NiONPs give promising antifungal activity against the causative species of mycotic keratitis, high penetration rates and good washing rates with no accumulation within mice tissues.

This was typically ensured by the statement of Lee et al. (2007), who suggested that nanoparticles are known to cross from the epidermis to the dermis poorly when topically applied to the skin whereas nanoparticle transport across the basal membrane is unidirectional whereby systemically administered nanoparticles do not cross the stratum basal and reach blood stream with very low concentrations. Findings of the present survey agreed with King \& Perry (2001), who reported low liver toxicity associated with topical treatment of mice with variable concentrations of NiONPs, as they recorded normal values of bilirubin, alkaline phosphatase, alanine aminotransferase, and aspartate aminotransferase, indicating normal liver function of treated animals.

\section{Conclusions}

In the present study, the single phase NiONPs was successfully biosynthesized extracellularly for the first time by the mycelial filtrate of the fungus $F$. verticillioides using $\mathrm{Ni}\left(\mathrm{NO}_{3}\right)_{2} \cdot 6 \mathrm{H}_{2} \mathrm{O}$ as starting material. XRD analysis shows that the produced NiONPs are in a cubical phase and measure about 8-17.7 $\mathrm{nm}$. TEM images shows that NiONPs have spherical shape and the average particle size found to be about 8.5-20.7 $\mathrm{nm}$. FTIR studies also confirm the formation of NiONPs. This method may be extending to the fungal biosynthesis of other metal oxides nanoparticles. Also, the biosynthesized NiONPs recorded a promising antifungal activity against fungal genera causing human mycotic keratitis at Tanta University hospital, with good washing out rates from experimental animal tissues. That opens a new trend for the topical use of these NiONPs preparations in the biomedical progress.

\section{References}

Abu El-Souod, S., Mahmoud, Y., El-Shourbagy, M. and El-Badry, A. (2013) Epidemiology of human mycotic keratitis in Tanta University Ophthalmology Hospital. Egyptian Journal of Experimental Biology (Botany), 9 , 65-74.

Al-Sehemi, A.G., Al-Shihri, A.S., Kalam, A., Du
G. and Ahmad, T. (2014) Microwave synthesis, optical properties and surface area studies of $\mathrm{NiO}$ nanoparticles. Journal of Molecular Structure 1058, 56-61.

Barakat, A., Al-Noaimi, M., Suleiman, M., Aldwayyan, A.S., Hammouti, B., Hadda, T.B., Haddad, S.F., Boshaala, A. and Warad, I. (2013) One step synthesis of $\mathrm{NiO}$ nanoparticles via solid-state thermal decomposition at low-temperature of novel aqua (2, 9-dimethyl-1, 10-phenanthroline) $\mathrm{NiCl}_{2}$ complex. International Journal of Molecular Sciences, 14, 23941-23954.

Barnett, H.L. and Hunter, B.B. (1998) "Illustrated Genera of Imperfect Fungi", American Phytopathological Society; $4^{\text {th }}$ ed.

Djalilian, A.R., Smith, J.A., Walsh, T.J., Malech, H.L. and Robinson, M.R. (2001) Keratitis caused by Candida glabrata in a patient with chronic granulomatous disease. American Journal of Ophthalmology, 132,782-783.

El-Badry, A.S. and Ali, S.S. (2015) Essential oils: A promising remedy against fungal and bacterial human keratitis. Egyptian Journal of Botany, 403-431.

El-Nahass, M., Emam-Ismail, M. and El-Hagary, M. (2015) Structural, optical and dispersion energy parameters of nickel oxide nanocrystalline thin films prepared by electron beam deposition technique. Journal of Alloys and Compounds, 646, 937-945.

Elsaesser, A. and Howard, C.V. (2012) Toxicology of nanoparticles. Advanced Drug Delivery Reviews 64,129-137.

Gondal, M., Saleh, T.A. and Drmosh, Q. (2012) Synthesis of nickel oxide nanoparticles using pulsed laser ablation in liquids and their optical characterization. Applied Surface Science, 258, 6982-6986.

Hunter, G.W. and Strickland, G.T. (2000) "Hunter's Tropical Medicine and Emerging Infectious Diseases",WB Saunders company.

Hussein, M. (2016) Silver tolerance and silver nanoparticle biosynthesis by Neoscytalidium novaehollandae and Trichoderma inhamatum. European Journal of Biological Research, 6, 28-35.

King, P.D. and Perry, M.C. (2001) Hepatotoxicity of chemotherapy. The Oncologist, 6,162-176.

Larone, D.H. (2011) "Medically Important Fungi: A Guide to Identification" Washington, DC: ASM Press.

Lasagna-Reeves, C., Gonzalez-Romero, D., Barria, M., Olmedo, I., Clos, A., Ramanujam, V.S., Urayama, A., Vergara, L., Kogan, M.J. and Soto, C. (2010) Bioaccumulation and toxicity of gold nanoparticles 
after repeated administration in mice. Biochemical and Biophysical Research Communications, 393, 649-655.

Leck, A., Thomas, P., Hagan, M., Kaliamurthy, J., Ackuaku, E., John, M., Newman, M., Codjoe, F., Opintan, J. and Kalavathy, C. (2002) Aetiology of suppurative corneal ulcers in Ghana and South India, and epidemiology of fungal keratitis. British Journal of Ophthalmology, 86, 1211-1215.

Lee, H.A., Imran, M., Monteiro-Riviere, N.A., Colvin, V.L., Yu, W.W. and Riviere, J.E. (2007) Biodistribution of quantum dot nanoparticles in perfused skin: evidence of coating dependency and periodicity in arterial extraction. Nano Letters, 7, 2865-2870.

Mahmoud, Y., El-Souod, S., El-Shourbagy, M. and ElBadry, A. (2004) Study on human corneal ulcers in Tanta University Ophthalmology Hospitals with special attention to mycotic keratitis. Bull. Fac. Sci. Assiut Univ. 33,1-15.

Mamuru, S.A., Bello, A.S. and Hamman, S.B. (2015) Annona squamosa leaf extract as an efficient bioreducing agent in the synthesis of chromium and nickel nanoparticles. International Journal of Applied Sciences and Biotechnology, 3,167-169.

Margo, C. and Brinser, J. (1987) "Microbiologic Diagnosis: Mycology: Laboratory Diagnosis in Ophthalmology", Burner, J. (Ed.), McMillan Press, New York, USA.

Moharram, A., Abdel-Kader, I., Al-Hussaini, K. and AlGhalibi, S. (1999) Studies on mycotic keratitis in Assuit Governorate. African Journal of Mycology and Biotechnology, 7,133-146.

Moubasher, A. (1993) "Soil Fungi in Qatar and Other Arab Countries", The Centre for Scientific and Applied Research, University of Qatar.

Pridham, T., Lindenfelser, L., Shotwell, O., Stodola, F., Benedict, R., Foley, C., Jacks, P., Zaumeyer, W., Perston, W. and Mitchell, J. (1956) Antibiotics against plant disease. I. Laboratory and green house survey. Phytopathol, 46, 568-575.

Qiao, H., Wei, Z., Yang, H., Zhu, L. and Yan, X. (2009) Preparation and characterization of $\mathrm{NiO}$ nanoparticles by anodic arc plasma method. Journal of Nanomaterials, $\mathbf{2}$.

Rahdar, A., Aliahmad, M. and Azizi, Y. (2015) NiO nanoparticles: Synthesis and characterization. Journal of Nanostructures, 5, 145-151.

Ramirez, C. (1982) "Manual and Atlas of the Penicillia" Elsevier Biomedical Press.
Raper, K.B. and Fennell, D.I. (1965) "The Genus Aspergillus". Williams and Wilkins, Philadelphia, 686 p.

Sagar, G. and Ashok, B. (2012) Green synthesis of silver nanoparticles using Aspergillus niger and its efficacy against human pathogens. Eur. J, Exp. Biol. 2,16541658 .

Salavati-Niasari, M., Mohandes, F., Davar, F., Mazaheri, M., Monemzadeh, M. and Yavarinia, N. (2009) Preparation of $\mathrm{NiO}$ nanoparticles from metal-organic frameworks via a solid-state decomposition route. Inorganica Chimica Acta, 362, 3691-3697.

Salvadori, M.R., Ando, R.A., Nascimento, C.A.O. and Corrêa, B. (2015) Extra and intracellular synthesis of nickel oxide nanoparticles mediated by dead fungal biomass. PloS one, 10(6), e0129799.

Shadomy, S., Espinel-Ingroff, A. and Cartwright, R. (1985) Laboratory studies with antifungal agents: susceptibility tests and bioassays. "Manual of Clinical Microbiology", $4^{\text {th }}$ ed. pp.991-999, American Society for Microbiology, Washington, DC.

Shi, C., Zhu, N., Cao, Y. and Wu, P. (2015) Biosynthesis of gold nanoparticles assisted by the intracellular protein extract of Pycnoporus sanguineus and its catalysis in degradation of 4-nitroaniline. Nanoscale Research Letters, 10,1-8.

Shukla, P. and Singh, P. (1997) Diagnosis of mycotic keratitis: an overview. "Advances in Medical Mycology". Lucknow, India: Council for Advances in Bioresources \& Evoker Research Perfecting Co:155-62.

Soni, N. and Prakash, S. (2012) Synthesis of gold nanoparticles by the fungus Aspergillus niger and its efficacy against mosquito larvae. Rep Parasitol. 2,1-7.

Sun, R.L., Jones, D.B. and Wilhelmus, K.R. (2007) Clinical characteristics and outcome of Candida keratitis. American Journal of Ophthalmology, 143,1043-1045.

Theivasanthi, T. and Alagar, M. (2012) Chemical capping synthesis of nickel oxide nanoparticles and their characterizations studies. arXiv preprint arXiv:1212.4595.

Thomas, P. and Kaliamurthy, J. (2013) Mycotic keratitis: epidemiology, diagnosis and management. Clinical Microbiology and Infection, 19, 210-220.

Wong, T.Y., Fong, K.S. and Tan, D.T. (1997) Clinical and microbial spectrum of fungal keratitis in Singapore: a 5-year retrospective study. International Ophthalmology, 21,127-130.

(Received 11/4/2017; accepted 4/5/2017) 


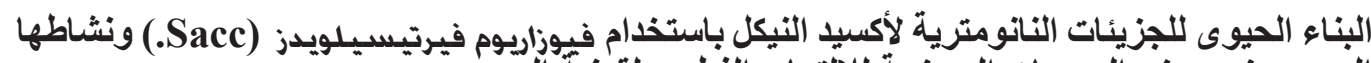 الخيوى ضد بعض المسبيات المرضية للأتّهاب الفطرى لقرنية العين

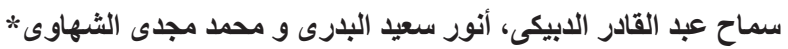

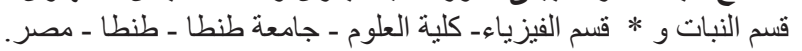

لقد تم خلال هذه الدراسة بناء جزيئات أكسيد النيكل متتاهية الصغر (نانو أكسيد النيكل)، حيوياً باستخدام

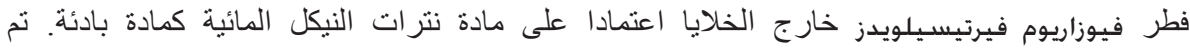

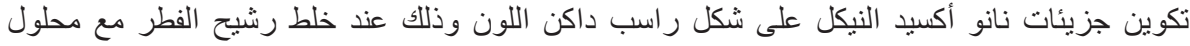

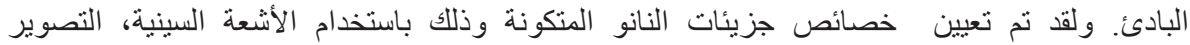
بالميكروسكوب الالكترونى النافذ والأشعة تحت الحمر اء. أكدت نتائج التحاليل باستخدام الأشعة تحت التحن

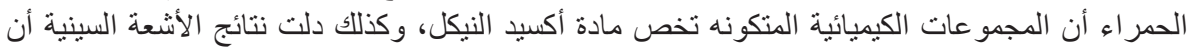

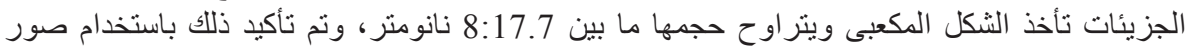

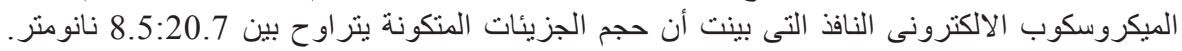

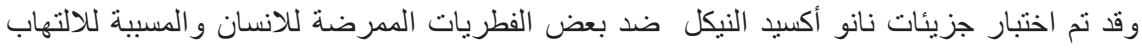

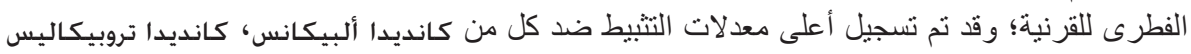

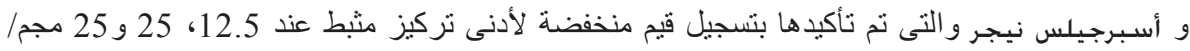
مل لكل منهم على التو الى ن.

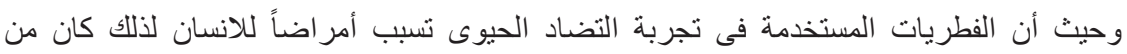

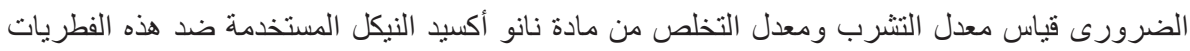

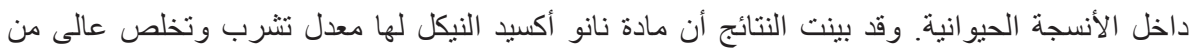

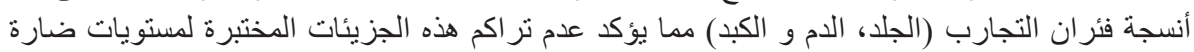

داخل الأنسجة. 